\title{
Fixed Points of $n$-Valued Multimaps of the Circle
}

\author{
by \\ Robert F. BROWN \\ Presented by Czestaw BESSAGA
}

\begin{abstract}
Summary. A multifunction $\phi: X \multimap Y$ is $n$-valued if $\phi(x)$ is an unordered subset of $n$ points of $Y$ for each $x \in X$. The (continuous) $n$-valued multimaps $\phi: S^{1} \multimap S^{1}$ are classified up to homotopy by an integer-valued degree. In the Nielsen fixed point theory of such multimaps, due to Schirmer, the Nielsen number $N(\phi)$ of an $n$-valued $\phi: S^{1} \multimap S^{1}$ of degree $d$ equals $|n-d|$ and $\phi$ is homotopic to an $n$-valued power map that has exactly $|n-d|$ fixed points. Thus the Wecken property, that Schirmer established for manifolds of dimension at least three, also holds for the circle. An $n$-valued multimap $\phi: S^{1} \multimap S^{1}$ of degree $d$ splits into $n$ selfmaps of $S^{1}$ if and only if $d$ is a multiple of $n$.
\end{abstract}

1. Introduction. A multifunction $\phi: X \multimap Y$ is a function such that $\phi(x)$ is a subset of $Y$ for each $x \in X$. For $S$ a subset of $Y$, the set $\phi^{-1}(S)$ consists of the points $x \in X$ such that $\phi(x) \subseteq S$, and the set $\phi_{+}^{-1}(S)$ consists of the points $x \in X$ such that $\phi(x) \cap S \neq \emptyset$. A multifunction $\phi$ is said to be upper semicontinuous (usc) if $U$ open in $Y$ implies $\phi^{-1}(U)$ is open in $X$. It is lower semicontinuous (lsc) if $U$ open in $Y$ implies $\phi_{+}^{-1}(U)$ is open in $X$. A multifunction that is both upper semicontinuous and lower semicontinuous is said to be continuous. Although the term multimap is sometimes used for a more general concept, in this paper it will mean a continuous multifunction. An $n$-valued multifunction $\phi: X \multimap Y$ is a function that assigns to each $x \in X$ an unordered subset of exactly $n$ points of $Y$. Thus an $n$-valued multimap is a continuous $n$-valued multifunction.

O'Neill [6] proved a version of the Lefschetz fixed point theorem for a large class of multimaps $\phi: X \multimap X$ of finite polyhedra that includes the $n$-valued multimaps. Multimaps in this class induce a vector space of endomorphisms of the homology of $X$. He proved that if any endomorphism

2000 Mathematics Subject Classification: Primary 55M20; Secondary 54C60, 55M25.

Key words and phrases: continuous multifunction, Wecken property, degree of $n$-valued map. 
has a nonzero Lefschetz number, then $\phi$ has a fixed point, that is, $x \in \phi(x)$ for some $x \in X$.

The Nielsen fixed point theory of $n$-valued multimaps was developed by Schirmer in a series of papers [7]-[9]. For $\phi: X \multimap X$ an $n$-valued multimap of a finite polyhedron, the Nielsen number $N(\phi)$ has the property that for any $n$-valued continuous homotopy $\Delta: X \times I \multimap X$ with $\Delta(x, 0)=\phi(x)$, the multimap $\psi: X \multimap X$ defined by $\psi(x)=\Delta(x, 1)$ has at least $N(\phi)$ fixed points.

The main result of [9] extended a celebrated theorem of Wecken [10] in the following way. If $\phi: X \multimap X$ is an $n$-valued multimap where $X$ is a compact triangulable manifold, with or without boundary, of dimension at least three, then there is an $n$-valued multimap $\psi: X \multimap X$ homotopic to $\phi$ such that $\psi$ has exactly $N(\phi)$ fixed points. As in the single-valued theory, we will refer to this property as the Wecken property for $n$-valued multimaps.

If $f_{0}, f_{1}, \ldots, f_{n-1}: X \rightarrow X$ are $n$ maps such that $j \neq k$ implies $f_{j}(x) \neq$ $f_{k}(x)$ for all $x \in X$ then

$$
\phi(x)=\left\{f_{0}(x), f_{1}(x), \ldots, f_{n-1}(x)\right\}
$$

defines an $n$-valued multimap $\phi: X \multimap X$ that is called split in [8]. Only two examples of nonsplit $n$-valued multimaps are included in Schirmer's papers; see page 75 of [7] and page 219 of [8]. The examples are of $n$-valued multimaps on the unit circle $S^{1}$ and thus the Wecken theorem of [9] does not apply to them. In both cases, the number of fixed points of the map $\phi$ that Schirmer defines is precisely $N(\phi)$, but there is no general such result about $n$-valued multimaps of the circle.

We recall that, in the single-valued case, among the manifolds only surfaces can fail to have the Wecken property that a selfmap $f: X \rightarrow X$ is homotopic to a map with exactly $N(f)$ fixed points [4], [5]. With regard to the 1-dimensional manifolds, the Wecken property holds for maps of the interval because they are all homotopic to a constant map. For $X=S^{1}$, there is the following well known argument that establishes the Wecken property for single-valued maps. By the classification theorem ([3, p. 39]), if $f: S^{1} \rightarrow S^{1}$ is of degree $d$, then $f$ is homotopic to the power map $\phi_{d}$ defined by viewing $S^{1}$ as the unit circle in the complex plane and setting $\phi_{d}(z)=z^{d}$. Thus $N(f)=N\left(\phi_{d}\right)$. It has long been known that $N\left(\phi_{d}\right)=|1-d|$ and clearly $\phi_{d}$ has $|1-d|$ fixed points except in the case $d=1$. Since $\phi_{1}$, the identity map, is homotopic to a fixed point free map, every selfmap $f$ on the circle is homotopic to a map with $N(f)$ fixed points.

The Wecken property is easily seen to hold for $n$-valued multimaps of the interval $I$, as follows. Let $\phi: I \multimap I$ be a multimap. Define $\Delta: I \times I \multimap I$ by $\Delta(s, t)=\phi(s t)$; then $\Delta$ is continuous by Theorems 1 and $1^{\prime}$ on page 113 of [2]. Thus $\phi$ is homotopic to the constant $n$-valued multimap $\kappa: I \multimap I$ 
defined by $\kappa(t)=\phi(0)$, which has $n$ fixed points, whereas $N(\kappa)=n$ by Corollary 7.3 of [8].

The purpose of this paper is to prove that the circle also has the Wecken property for $n$-valued multimaps. In outline, the argument follows that of the single-valued setting, but there are several significant issues that must be addressed in the $n$-valued case. In Section 2, we extend the definition of the degree of a selfmap of the circle to define the degree of an $n$-valued multimap of the circle and we discuss its properties. Section 3 introduces a collection of $n$-valued multimaps we call $n$-valued power maps $\phi_{n, d}: S^{1} \multimap S^{1}$ and we extend the classification theorem by proving that an $n$-valued multimap $\phi: S^{1} \multimap S^{1}$ of degree $d$ is homotopic to $\phi_{n, d}$. We prove in Section 4 that $\phi_{n, d}$ has $|n-d|$ fixed points if $n \neq d$ and then that $N\left(\phi_{n, d}\right)=|n-d|$ for all $n$ and $d$. In Section 5, the Wecken property for $n$-valued multimaps of the circle is easily seen to follow from the previous results. Moreover, we characterize the split $n$-valued multimaps of the circle: an $n$-valued multimap is split if and only if its degree is a multiple of $n$.

2. The degree of an $n$-valued multimap of the circle. We begin with some general properties of $n$-valued multimaps. The following result is a special case of a theorem of O'Neill [6] but, according to [9], it was essentially known much earlier [1].

Lemma 2.1 (Splitting Lemma). Let $\phi: X \multimap Y$ be an $n$-valued multimap and let

$$
\Gamma_{\phi}=\{(x, y) \in X \times Y: y \in \phi(x)\}
$$

be the graph of $\phi$. The map $p_{1}: \Gamma_{\phi} \rightarrow X$ defined by $p_{1}(x, y)=x$ is a covering space. It follows that if $X$ is simply connected, then any n-valued multimap $\phi: X \multimap Y$ is split.

TheOREM 2.1. Let $\Delta: X \times I \multimap Y$ be an n-valued homotopy; write $\Delta=\left\{\delta^{t}: X \multimap Y\right\}$. If $\delta^{0}$ is split, so also is $\Delta$. Thus an n-valued multimap homotopic to a split n-valued multimap is also split.

Proof. Write $\delta^{0}=\left\{f_{0}^{0}, f_{1}^{0}, \ldots, f_{n-1}^{0}\right\}$ where $f_{j}^{0}: X \rightarrow Y$. Define

$$
\widehat{f}_{0}^{0}: X \times\{0\} \rightarrow \Gamma_{\Delta} \subseteq(X \times I) \times Y
$$

by $\widehat{f}_{0}^{0}(x, 0)=\left((x, 0), f_{0}^{0}(x)\right)$. Since $p_{1}: \Gamma_{\Delta} \rightarrow X \times I$ is a covering space by Lemma 2.1, by the covering homotopy property there is a map $\widehat{f}_{0}: X \times$ $I \rightarrow \Gamma_{\Delta}$ such that $p_{1} \widehat{f_{0}}$ is the identity map of $X \times I$. Let $p_{2}: \Gamma_{\Delta} \rightarrow Y$ be projection. Then $p_{2} \widehat{f}_{0}(x, t) \in \delta^{t}(x)$ so $p_{2} \widehat{f}_{0}$ is a selection for $\Delta$ and we can write $\Delta=\left\{p_{2} \widehat{f}_{0}, \Delta^{\prime}\right\}$ where $\Delta^{\prime}: X \multimap Y$ is an $(n-1)$-valued homotopy $\Delta^{\prime}=\left\{\delta^{\prime t}\right\}$ with $\delta^{\prime 0}=\left\{f_{1}^{0}, \ldots, f_{n-1}^{0}\right\}$. Repeated application of the covering homotopy property produces a splitting $\Delta=\left\{p_{2} \widehat{f}_{0}, p_{2} \widehat{f}_{1}, \ldots, p_{2} \widehat{f}_{n-1}\right\}$. If an 
$n$-valued multimap $\psi: X \multimap Y$ is homotopic to a split $n$-valued multimap $\phi=\left\{f_{0}, \ldots, f_{n-1}\right\}$ by a homotopy $\Delta$ with $\delta^{0}=\phi$ and $\delta^{1}=\psi$, then $\psi=$ $\left\{f_{0}^{1}, \ldots, f_{n-1}^{1}\right\}$ where $f_{j}^{1}(x)=p_{2} \widehat{f}_{j}(x, 1)$.

Now we turn our attention to the circle and let $p: \mathbb{R} \rightarrow S^{1}$ be the universal covering space where $p(t)=e^{i 2 \pi t}$. We will denote points of the circle by $p(t)$ for $0 \leq t<1$. Let $\phi: S^{1} \multimap S^{1}$ be an $n$-valued multimap. Then the $n$-valued function $\phi p: I \multimap S^{1}$ is continuous by Theorems 1 and $1^{\prime}$ on page 133 of [2]. Therefore $\phi p$ is split and, using the ordering on $S^{1}$ imposed by $p$ from the ordering of $\mathbb{R}$, we write $\phi p=\left\{f_{0}, f_{1}, \ldots, f_{n-1}\right\}$ where the maps $f_{j}: I \rightarrow S^{1}$ have the property $f_{j}(0)=p\left(t_{j}\right)$ for $0 \leq t_{0}<t_{1}<\cdots<t_{n-1}<1$. Let $\widetilde{f}_{j}: I \rightarrow \mathbb{R}$ be the lift of $f_{j}$ such that $\tilde{f}_{j}(0)=t_{j}$. We note that if $0 \leq j<k \leq$ $n-1$, then $\tilde{f}_{j}(t)<\widetilde{f}_{k}(t)$ for all $t \in I$ because $f_{j}(p(t)) \neq f_{k}(p(t))$.

Since $\phi$ is well defined, the sets $\phi p(0)$ and $\phi p(1)$ must be identical. Consequently, $\widetilde{f}_{0}(1)=v+t_{J}$ for some integers $v, J$ where $0 \leq J \leq n-1$. We define $\operatorname{Deg}(\phi)$, the degree of the $n$-valued multimap $\phi: S^{1} \multimap S^{1}$, by

$$
\operatorname{Deg}(\phi)=n v+J .
$$

The degree can be defined just in terms of $\widetilde{f}_{0}(1)$ because that value determines $\widetilde{f}_{j}(1)$ for all $j$, as the next result demonstrates.

Lemma 2.2. Let $\phi: S^{1} \multimap S^{1}$ be an n-valued multimap of degree $\operatorname{Deg}(\phi)$ $=n v+J$. For $\phi p=\left\{f_{0}, f_{1}, \ldots, f_{n-1}\right\}$ where the maps $f_{j}: I \rightarrow S^{1}$ have the property $f_{j}(0)=p\left(t_{j}\right)$ with $0 \leq t_{0}<t_{1}<\cdots<t_{n-1}<1$ and $\tilde{f}_{j}$ the lift of $f_{j}$ such that $\widetilde{f}_{j}(0)=t_{j}$, we have $\widetilde{f}_{n-1}(1)-\widetilde{f}_{0}(1)<1$. Therefore, $\widetilde{f}_{j}(1)=v+t_{J+j}$ for $j=0, \ldots,(n-1)-J$ and, if $J \geq 1$, then $\widetilde{f}_{j}(1)=v+1+t_{j-(n-J)}$ for $j=n-J, \ldots, n-1$.

Proof. Define $F: I \rightarrow \mathbb{R}$ by $F(t)=\widetilde{f}_{n-1}(t)-\widetilde{f}_{0}(t)$. Then $F(0)=t_{n-1}-$ $t_{0}<1$. If $F(1)>1$, then $F\left(t^{*}\right)=1$ for some $t^{*} \in(0,1)$ and thus $\widetilde{f}_{n-1}\left(t^{*}\right)=$ $\widetilde{f}_{0}\left(t^{*}\right)+1$. But $\widetilde{f}_{j}$ is a lift of $f_{j}$ so we would have

$$
p \widetilde{f}_{n-1}\left(t^{*}\right)=f_{n-1}\left(p\left(t^{*}\right)\right)=p\left(\widetilde{f}_{0}\left(t^{*}\right)+1\right)=p\left(\widetilde{f}_{0}\left(t^{*}\right)\right)=f_{0}\left(p\left(t^{*}\right)\right)
$$

contrary to the definition of a splitting. The formulas for the $\widetilde{f}_{j}(1)$ then follow because $\widetilde{f}_{0}(t)<\widetilde{f}_{1}(t)<\cdots<\widetilde{f}_{n-1}(t)$ for all $t \in I$.

The fact that this definition of degree agrees with the classical definition when $n=1$ is a special case of the following result.

TheOREM 2.2. If $\phi: S^{1} \multimap S^{1}$ is a split n-valued multimap, then $\operatorname{Deg}(\phi)$ equals $n$ times the classical degree of the maps in the splitting.

Proof. Write $\phi=\left\{f_{0}, f_{1}, \ldots, f_{n-1}\right\}$ where $f_{j}(p(0))=p\left(t_{j}\right)$ and $0 \leq t_{0}<$ $t_{1}<\cdots<t_{n-1}<1$. Let $\widetilde{f}_{j}: I \rightarrow \mathbb{R}$ be the lift of $f_{j} p: I \rightarrow S^{1}$ such that 
$\widetilde{f}_{j}(0)=t_{j}$. Since $f_{0}: S^{1} \rightarrow S^{1}$, we have $\widetilde{f}_{0}(1)=v+\widetilde{f}_{0}(0)=v+t_{0}$ for some integer $v$ and thus $\operatorname{Deg}(\phi)=n v$. Moreover, Lemma 2.2 implies that $\widetilde{f}_{j}(1)=v+t_{j}$ for $j=0, \ldots, n-1$. On the other hand, by the argument on page 39 of [3], each map $f_{j}$ is homotopic to the power map $\phi_{v}: S^{1} \rightarrow S^{1}$ and therefore it is of classical degree $\operatorname{deg}\left(f_{j}\right)=v$, so $\operatorname{Deg}(\phi)=n \operatorname{deg}\left(f_{j}\right)$.

THEOREM 2.3. If n-valued multimaps $\phi, \psi: S^{1} \multimap S^{1}$ are homotopic, then $\operatorname{Deg}(\phi)=\operatorname{Deg}(\psi)$.

Proof. Let $\Delta=\left\{\delta^{t}\right\}: S^{1} \multimap S^{1}$ be an $n$-valued homotopy with $\phi=\delta^{0}$ and $\psi=\delta^{1}$. We will show that there exists $\varepsilon>0$ such that if $\left|t-t^{\prime}\right|<\varepsilon$, then $\operatorname{Deg}\left(\delta^{t}\right)=\operatorname{Deg}\left(\delta^{t^{\prime}}\right)$, that is, the degree is locally constant. Since the degree is integer-valued, that will imply that it is constant and therefore $\operatorname{Deg}(\phi)=\operatorname{Deg}(\psi)$. Write $\delta^{t} p=\left\{f_{0}^{t}, f_{1}^{t}, \ldots, f_{n-1}^{t}\right\}$ where $f_{j}^{t}(0)=p\left(t_{j}\right)$ for $0 \leq t_{0}<t_{1}<\cdots<t_{n-1}<1$. Let $\widetilde{f}_{j}^{t}: I \rightarrow \mathbb{R}$ be the lift of $f_{j}^{t}$ such that $\tilde{f}_{j}^{t}(0)=t_{j}$. We use the corresponding notation for $\delta^{t^{\prime}}$. If $\widetilde{f}_{j}^{t}(1)=v+t_{J}$ where $t_{J}>0$ then, by the continuity of $\Delta$, if $\varepsilon>0$ is small enough, $\left|t-t^{\prime}\right|<\varepsilon$ implies that $\widetilde{f}_{j}^{t^{\prime}}(1)=v+t_{J}^{\prime}$ where $t_{J}^{\prime}>0$ and therefore

$$
\operatorname{Deg}\left(\delta^{t}\right)=\operatorname{Deg}\left(\delta^{t^{\prime}}\right)=n v+J .
$$

If $\widetilde{f}_{0}^{t}(1)=v=v+0$, that means $t_{0}=0$ so

$$
\widetilde{f}_{j}^{t}(1)=v+t_{j}=v+\widetilde{f}_{j}^{t}(0)
$$

for all $j$ by Lemma 2.2. Therefore, the $f_{j}^{t}: S^{1} \rightarrow S^{1}$ defined by $f_{j}^{t}(p(s))=$ $p \widetilde{f}_{j}^{t}(s)$ splits $\delta^{t}$ and thus $\operatorname{Deg}\left(\delta^{t}\right)=n \cdot \operatorname{deg}\left(f_{0}^{t}\right)$ by Theorem 2.2. Since $\delta^{t^{\prime}}$ is homotopic to $\delta^{t}$, Theorem 2.1 shows that $\delta^{t^{\prime}}$ is also split and $f_{0}^{t^{\prime}}$ is homotopic to $f_{0}^{t}$ so, for the classical degrees, $\operatorname{deg}\left(f_{0}^{t}\right)=\operatorname{deg}\left(f_{0}^{t^{\prime}}\right)$ and thus $\operatorname{Deg}\left(\delta^{t}\right)=$ $\operatorname{Deg}\left(\delta^{t^{\prime}}\right)$.

3. The classification theorem. For integers $d$ and $n \geq 1$, we define the $n$-valued multimap we call the $n$-valued power map $\phi_{n, d}: S^{1} \multimap S^{1}$ by

$$
\phi_{n, d}(p(t))=\left\{p\left(\frac{d}{n} t\right), p\left(\frac{d}{n} t+\frac{1}{n}\right), \ldots, p\left(\frac{d}{n} t+\frac{n-1}{n}\right)\right\} .
$$

Since

$$
\phi_{1, d}(p(t))=p(d t)=e^{i 2 \pi d t}=\left(e^{i 2 \pi t}\right)^{d}=(p(t))^{d},
$$

we see that $\phi_{1, d}=\phi_{d}$. The example on page 75 of [7] is $\phi_{2,1}$ and the example on page 219 of [8] is $\phi_{2,-1}$.

Lemma 3.1. The degree of $\phi_{n, d}$ is $d$. 
Proof. We see that $\phi_{n, d} p=\left(p \widetilde{f}_{0}, \ldots, p \widetilde{f}_{n-1}\right)$ where $\tilde{f}_{j}(t)=d t / n+j / n$ so $\tilde{f}_{j}(0)=j / n=t_{j}$. Write $d=n v+J$ where $0 \leq J \leq n-1$. Then

$$
\widetilde{f}_{0}(1)=\frac{d}{n}=v+\frac{J}{n}=v+\widetilde{f}_{J}(0)=v+t_{J}
$$

so, from the definition, $\operatorname{Deg}\left(\phi_{n, d}\right)=n v+J=d$.

Theorem 3.1 (Classification Theorem). If $\phi: S^{1} \multimap S^{1}$ is an n-valued multimap of degree $d$, then $\phi$ is homotopic to $\phi_{n, d}$.

Proof. We again write $\phi p=\left\{f_{0}, f_{1}, \ldots, f_{n-1}\right\}: I \multimap S^{1}$ and lift $f_{j}$ to $\tilde{f}_{j}: I \rightarrow \mathbb{R}$ such that $\tilde{f}_{j}(0)=t_{j}$ where $f_{j}(0)=p\left(t_{j}\right)$ and $0 \leq t_{0}<t_{1}<\cdots<$ $t_{n-1}<1$. Define maps $\widetilde{h}_{j}^{s}: I \times I \rightarrow \mathbb{R}$ by

$$
\widetilde{h}_{j}^{s}(t)=s\left(\frac{d}{n} t+j\right)+(1-s) \tilde{f}_{j}(t) .
$$

Then it is clear that $j<k$ implies $\widetilde{h}_{j}^{s}(t)<\widetilde{h}_{k}^{s}(t)$ for all $s, t \in I$. Write $\operatorname{Deg}(\phi)=d=n v+J$ where $0 \leq J \leq n-1$. Suppose $0 \leq j \leq(n-1)-J$. Then, by Lemma 2.2 , we have $\widetilde{h}_{j}^{s}(1)-\widetilde{h}_{J+j}^{s}(0)=v$. For $J \geq 1$ and $n-J \leq j \leq n-1$, Lemma 2.2 implies that $\widetilde{h}_{j}^{s}(1)-\widetilde{h}_{j-(n-J)}^{s}(0)=v+1$. Thus, for all $s \in I$, the sets $\left\{p \widetilde{h}_{j}^{s}(0)\right\}$ and $\left\{p \widetilde{h}_{j}^{s}(1)\right\}$ are identical. Therefore, setting

$$
\Delta(p(t), s)=\left\{p \widetilde{h}_{0}^{s}(t), p \widetilde{h}_{1}^{s}(t), \ldots, p \widetilde{h}_{n-1}^{s}(t)\right\}
$$

we obtain a homotopy $\Delta: S^{1} \times I \multimap S^{1}$ between $\phi$ and $\phi_{n, d}$.

\section{Properties of the $n$-valued power maps}

THEOREM 4.1. If $n \neq d$, then the $n$-valued power map $\phi_{n, d}$ has $|n-d|$ fixed points, each of nonzero index, and no two fixed points are in the same fixed point class, therefore $N\left(\phi_{n, d}\right)=|n-d|$.

Proof. If $p(t) \in \phi_{n, d}(p(t))$ for some $t$ such that $0 \leq t<1$ then, for some $j=0,1, \ldots, n-1$, we have

$$
p\left(\frac{d}{n} t+\frac{j}{n}\right)=p(t)
$$

and therefore

$$
\frac{d}{n} t+\frac{j}{n}-t=\frac{(d-n) t}{n}+\frac{j}{n}=r
$$

for some integer $r$. Since $n \neq d$, the possible solutions are of the form

$$
t=\frac{n r-j}{d-n}
$$

where $r$ and $j$ are integers and $0 \leq j \leq n-1$. We require that $0 \leq t<1$ so if $d-n>0$, then $0 \leq n r-j<d-n$, whereas if $d-n<0$, then $0 \geq n r-j>d-n$. In either case, there are $|d-n|$ such integers and we 
conclude that $\phi_{n, d}$ has $|d-n|$ fixed points. Each of the $|n-d|$ fixed points of $\phi_{n, d}$ is transversal and therefore of index \pm 1 (see page 210 of [8]).

It remains to prove that no two of the fixed points of $\phi_{n, d}$ are equivalent in the sense of [8]. Noting that the fixed points are of the form $p\left(\frac{n r-j}{d-n}\right)$, we will make use of the fact that

$$
\frac{d}{n}\left(\frac{n r-j}{d-n}\right)+\frac{j}{n}=r+\frac{n r-j}{d-n} .
$$

For $k=0,1$, let

$$
x_{k}=p\left(\frac{n r_{k}-j_{k}}{d-n}\right)=p\left(\widetilde{x}_{k}\right)
$$

be two fixed points of $\phi_{n, d}$ and let $a: I \rightarrow S^{1}$ be a path such that $a(k)=x_{k}$. Let $\widetilde{a}: I \rightarrow \mathbb{R}$ be the lift of $a$ such that $\widetilde{a}(0)=\widetilde{x}_{0} \in[0,1)$. Since $a=p \widetilde{a}$, we can write

$$
\begin{aligned}
\phi_{n, d} a(t) & =\phi_{n, d} p(\widetilde{a}(t)) \\
& =\left\{p\left(\frac{d}{n} \widetilde{a}(t)\right), p\left(\frac{d}{n} \widetilde{a}(t)+\frac{1}{n}\right), \ldots, p\left(\frac{d}{n} \widetilde{a}(t)+\frac{n-1}{n}\right)\right\} \\
& =\left\{g_{0}(t), g_{1}(t), \ldots, g_{n-1}(t)\right\},
\end{aligned}
$$

a split multimap. The fixed points $x_{0}$ and $x_{1}$ are in the same fixed point class if there exists a path $a$ connecting them and some $j^{*}$ with $0 \leq j^{*} \leq n-1$ such that $g_{j^{*}}\left(x_{k}\right)=x_{k}$ for $k=0,1$ and the paths $a, g_{j^{*}}: I \rightarrow S^{1}$ are homotopic relative to the endpoints (see [8, p. 214]).

We claim that the condition $g_{j^{*}}\left(x_{0}\right)=x_{0}$ implies that $j^{*}=j_{0}$. To prove it, we note that since $a(0)=\widetilde{x}_{0}$, it follows that

$$
p\left(\frac{d}{n}\left(\frac{n r_{0}-j_{0}}{d-n}\right)+\frac{j^{*}}{n}\right)=p\left(\frac{n r_{0}-j_{0}}{d-n}\right)
$$

and therefore

$$
\frac{d}{n}\left(\frac{n r_{0}-j_{0}}{d-n}\right)+\frac{j^{*}}{n}=\frac{n r_{0}-j_{0}}{d-n}+m
$$

for some integer $m$, which implies

so

$$
r_{0}+\frac{n r_{0}-j_{0}}{d-n}+\frac{j^{*}-j_{0}}{n}=\frac{n r_{0}-j_{0}}{d-n}+m
$$

$$
\frac{j^{*}-j_{0}}{n}=m-r_{0}
$$

an integer. But $0 \leq j^{*}, j_{0} \leq n-1$ and therefore $j^{*}=j_{0}$. This establishes the claim and we write $g=g_{j^{*}}=g_{j_{0}}: I \rightarrow S^{1}$ as the path from $x_{0}$ to $x_{1}$ that is homotopic to $a$ relative to the endpoints.

Let $\widetilde{g}: I \rightarrow \mathbb{R}$ be the lift of $g$ defined by

$$
\widetilde{g}(t)=\frac{d}{n} \widetilde{a}(t)+\frac{j_{0}}{n}-r_{0} .
$$


Then $\widetilde{g}(0)=\widetilde{x}_{0}=\widetilde{a}(0)$. Since $a g^{-1}$ is a contractible loop, its lift $\widetilde{a}^{-1}$ is also a loop and thus $\widetilde{g}(1)=\widetilde{a}(1)=\widetilde{x}_{1}+q$ for some integer $q$. Now

$$
\begin{aligned}
\widetilde{g}(1) & =\frac{d}{n}\left(\frac{n r_{1}-j_{1}}{d-n}+q\right)+\frac{j_{0}}{n}-r_{0} \\
& =r_{1}+\frac{n r_{1}-j_{1}}{d-n}+\frac{j_{0}-j_{1}}{n}+\frac{d}{n} q-r_{0},
\end{aligned}
$$

which implies that

$$
q=r_{1}-r_{0}+\frac{j_{0}-j_{1}}{n}+\frac{d}{n} q
$$

and thus that

$$
q=\frac{n r_{1}-j_{1}}{d-n}-\frac{n r_{0}-j_{0}}{d-n}=\widetilde{x}_{1}-\widetilde{x}_{0} .
$$

Then $0 \leq \widetilde{x}_{0}, \widetilde{x}_{1}<1$ implies that $q=0$ so $\widetilde{x}_{0}=\widetilde{x}_{1}$ and therefore $x_{0}=x_{1}$. We conclude that no two distinct fixed points of $\phi_{n, d}$ are in the same fixed point class.

\section{The Wecken property and split multimaps}

Theorem 5.1 (The Wecken Property). The circle has the Wecken property for $n$-valued multimaps because, if $\phi: S^{1} \multimap S^{1}$ is an n-valued multimap of degree $d$, then $N(\phi)=|n-d|$ and there is an n-valued multimap homotopic to $\phi$ that has exactly $|n-d|$ fixed points.

Proof. By Theorem 3.1, $\phi$ is homotopic to $\phi_{n, d}$ so $N(\phi)=N\left(\phi_{n, d}\right)$ by Theorem 6.5 of [8]. If $d=n$, then $\phi$ is homotopic to $\phi_{n, n}$. Choose $0<\varepsilon<1 / n$ and define $\Delta: S^{1} \times I \multimap S^{1}$ by

$$
\Delta(p(t), s)=\left\{p(t+s \varepsilon), p\left(t+s \varepsilon+\frac{1}{n}\right), \ldots, p\left(t+s \varepsilon+\frac{n-1}{n}\right)\right\} .
$$

Then $\phi_{n, n}$ is homotopic by $\Delta$ to a fixed point free multimap. Furthermore, $N(\phi)=N\left(\phi_{n, n}\right)=0$. If $n \neq d$, then Theorem 4.1 completes the proof because $N(\phi)=N\left(\phi_{n, d}\right)=|n-d|$ and $\phi_{n, d}$ has $|n-d|$ fixed points.

THEOREM 5.2. The power map $\phi_{n, d}$ is split if and only if $d$ is a multiple of $n$.

Proof. The graph of $\phi_{n, d}$ is

$$
\Gamma_{\phi_{n, d}}=\left\{\left(p(t), p\left(\frac{d}{n} t+\frac{j}{n}\right)\right): t \in \mathbb{R}, j=0,1, \ldots, n-1\right\} .
$$

For $j \in\{0,1, \ldots, n-1\}$ define $\gamma_{j}: I \rightarrow \Gamma_{\phi_{n, d}}$ by

$$
\gamma_{j}(t)=\left(p(t), p\left(\frac{d}{n} t+\frac{j}{n}\right)\right) \text {. }
$$


Let $\Gamma^{j} \subseteq \Gamma_{\phi_{n, d}}$ be the component of the graph containing $(p(0), p(j / n))$. Then $p_{1 j}: \Gamma^{j} \rightarrow S^{1}$, the restriction of $p_{1}$ to $\Gamma^{j}$, is a covering space and $\gamma_{j}$ is a path in $\Gamma^{j}$ from $(p(0), p(j / n))$ to $(p(0), p(d / n+j / n))$. Write $d=n v+J$ where $0 \leq J \leq n-1$. Then

$$
p\left(\frac{d}{n}+\frac{j}{n}\right)=p\left(\frac{r n+J+j}{n}\right)=p\left(\frac{J+j}{n}\right)
$$

tells us that $p(j / n)=p(d / n+j / n)$ and thus $\gamma_{j}(0)=\gamma_{j}(1)$ if, and only if, $J=0$, that is, if and only if $d$ is a multiple of $n$. If $d$ is not a multiple of $n$, then we have shown that the fiber of every covering space $p_{1 j}: \Gamma^{j} \rightarrow S^{1}$ obtained by restricting $p_{1}$ to a component of $\Gamma_{\phi_{n, d}}$ contains at least two points. If $\phi_{n, d}$ were split, it would have a selection, that is, there would be a map $f: S^{1} \rightarrow S^{1}$ such that $f(p(t)) \in \phi_{n, d}(p(t))$ for each $t \in I$. In particular, $(p(0), f(p(0))) \in \Gamma^{j}$ for some $j$ and thus $\sigma: S^{1} \rightarrow \Gamma^{j}$ defined by $\sigma(p(t))=\left(p(t), f(p(t))\right.$ is a cross-section of the covering space $p_{1 j}: \Gamma^{j} \rightarrow$ $S^{1}$, that is, $p_{1 j} \sigma$ is the identity map of $S^{1}$. Thus $p_{1 j} \sigma$ would induce the identity isomorphism on the fundamental group of $S^{1}$. But that is impossible because the index of the image of the homomorphism induced by $p_{1 j}$ in that fundamental group equals the cardinality of the fiber of the covering space, which is greater than one. On the other hand, if $d$ is a multiple of $n$, then $\phi_{n, d}$ splits as $\phi_{n, d}=\left\{f_{0}, f_{1}, \ldots, f_{n-1}\right\}$ where the map $f_{j}: S^{1} \rightarrow S^{1}$ is defined by $f_{j}(p(t))=p(d t / n+j / n)$.

Corollary 5.1. If $\phi: S^{1} \multimap S^{1}$ is an n-valued multimap of degree $d$, then $\phi$ is split if and only if $d$ is a multiple of $n$.

Proof. By Theorem 3.1, $\phi$ is homotopic to $\phi_{n, d}$. Therefore, by Theorem 2.1, $\phi$ is split if and only if $\phi_{n, d}$ is split, which, by Theorem 5.2, occurs if and only if $d$ is a multiple of $n$.

\section{References}

[1] S. Banach und S. Mazur, Über mehrdeutige stetige Abbildungen, Studia Math. 5 (1934), 174-178.

[2] C. Berge, Topological Spaces, Oliver \& Boyd, 1963.

[3] S. Hu, Homotopy Theory, Academic Press, 1959.

[4] B. Jiang, On the least number of fixed points, Amer. J. Math. 102 (1980), 749-763.

[5] —, Fixed points and braids, II, Math. Ann. 272 (1985), 249-256.

[6] B. O'Neill, Induced homology homomorphisms for set-valued maps, Pacific J. Math. 7 (1957), 1179-1184.

[7] H. Schirmer, Fix-finite approximations of n-valued multifunctions, Fund. Math. 121 (1984), 73-80.

[8] -, An index and Nielsen number for n-valued multifunctions, ibid. 124 (1984), $207-219$. 
[9] H. Schirmer, A minimum theorem for n-valued multifunctions, ibid. 126 (1985), 83-92.

[10] F. Wecken, Fixpunktklassen, III, Math. Ann. 118 (1942), 544-577.

Robert F. Brown

Department of Mathematics

University of California

Los Angeles, CA 90095-1555, U.S.A.

E-mail: rfb@math.ucla.edu

Received September 25, 2006

$(7553)$ 